\title{
COMPARING Optical PACKeT SWITCHING AND OPTICAL BURST SWITCHING
}

\author{
Sajjad Waheed \\ Assistant Professor, Department of Information and \\ Communication Technology, Mawlana Bhashani Science \\ and Technology University, Tangail \\ Email: sajad302@yahoo.com
}

\begin{abstract}
The switching is an important part of the telecommunication networks over the years for transferring and handling voice and data through the traditional switching nodes existing in the telecommunication networks. Various developments were seen in the recent years which resulted in increasing data transfer capabilities of the transmission networks all over the world, however the switching speed and techniques are still remained unchanged in most of the cases that neither match the data transfer capacities nor the expanding demand of the networks in the near future. Some switching technologies are still in the developing stage, of them two are more obvious: Optical Packet Switching (OPS) and Optical Burst Switching (OBS). In this paper, I tried to compare different paradigm of these two main developments, keeping in mind that the OPS would see rapid development in the near future than the OBS, though OBS would the ultimate solution of switching for the bulk volume of telephone and data traffic
\end{abstract}

Keywords: Optical Packet Switching, Optical Burst Switching, Bulk Volume, Data Traffic and Wavedivision Multiplexing

\section{Introduction}

Wavelength-Division multiplexing (WDM) [1] is a promising technique to utilize the enormous bandwidth of the optical fiber. A fundamental requirement in fiber-optic communication is that these channels operate at different wavelengths can be independently modulated to accommodate dissimilar data formats. WDM utilizes the huge bandwidth ( $50 \mathrm{THz}$ ) of a single-mode optical fiber while providing channels whose bandwidths (1-10 $\mathrm{Gb} / \mathrm{s}$ ) are compatible with current electronic processing speeds. The use of wavelengths to route data is referred to as wavelength routing, and a network which employs this technique is Date of submission : 03.02.2010 Date of acceptance : 06.01.2011 known as a wavelength-routed network [2]. A network consists of wavelength-routing switches (or routing nodes) are interconnected by optical fibers of which some of those routing nodes (referred to as crossconnects also) are attached to access stations where data from several end-users could be multiplexed on to a single WDM channel. An access station also provides optical-toelectronic $(\mathrm{O} / \mathrm{E})$ conversion and vice versa to interface the optical network with conventional electronic equipment. A wavelength-routed network which carries data from one access station to another without any intermediate $\mathrm{O} / \mathrm{E}$ conversion is referred to as an all-optical wavelength-routed network.

The wavelength routing in the said manner are done through Optical Packet Switching (OPS) and Optical Burst Switching (OBS). According the contemporary works discussed in various research articles, every researcher is of the opinion that WDM shall be the future solution to the explosive growth of the Internet, hence compatible switching like optical packet or optical burst switches will be required for switching optical traffic.

Out of different developed techniques for the transfer of data over WDM, broadcast-andselect, wavelength routing, optical packet switching, and optical burst switching are mentionable. Wavelength routing networks are already in use. Prototypes of broadcastand-select networks have been developed. However, optical packet switching and optical burst switching are still in the research phase.

\section{Optical Packet Switches}

The main purpose of the optical switches is to connect two ends of an optical transmission line. The switches are as such that the user 
data remains as an optical signal during its entire travel duration. The aim of such a switch is to make signals all optical from the beginning to the end, that is, a signal is never converted from optical to electrical or vise versa, thus setting up an end-to-end connection and reserve resources to transfer of traffic through an optical transport network for the burst of the signal in question.

Optical switching is suitable for ATM and IP traffics. However, some technical difficulties have barred optical packet switching from becoming more successful, and much works were not done with the IP packets transfer techniques. On the other hand, more research were done in the optical burst switching for which it is becoming a promising solution for the WDM techniques.

In order to transfer data from one access station to another, a connection needs to be set up at the optical layer. Optical packet network consists of optical packet switches interconnected with fibers running WDM. This operation is performed by determining a path (route) in the network connecting the source station to the destination station and by allocating a common free (or idle) wavelength on all of the fiber links in the path. Such an alloptical path is referred to as a lightpath or a clear channel. The entire bandwidth available on this lightpath is allocated to the connection during its holding time during which the corresponding wavelength cannot be allocated to any other connection.

\section{Construction of Optical Packet Switches}

The basic structure of the optical packet switch contains an input interface, a switching fabric, an output interface, and a control unit. The input interface receives packets, aligned those packets, extract header information and remove header. The switch fabric is performs optical switching. The output interface regenerates optical signals and put header back to the packet. The control unit controls whole affair. Optical packet switches are typically designed for fixed-size packets.

\section{Techniques of Packet Processing and Coding}

Unlike the classical switches, the WDM optical packet switch receives packets at the input interface. The header is separated from the payload. The header is then converted into an electric signal and processed electronically. However, the payload remains an optical signal and passes through the switching fabric. After the switching is done, both the header and payload are put together and the signal is converted into optical one at the output interface.

There are many packet coding techniques, of which three are of basic types: bit serial, bit parallel, and out-of-band signaling [3].

Bit serial coding can be achieved through any of the mentioned techniques: optical codedivision multiplexing (OCDM), optical pulse interval, or mixed-rate techniques. Each bit has its own routing information in OCDM. For the other two techniques, multiple bits are arranged in such a manner that they form a payload and header separately. In th e optical pulse interval, both the packet header and the payload pass the switch at the same rate. However, the header is processed electronically which has a lower transmission rate than the payload.

Bit parallel coding is implemented with multiple bits which are transmitted in parallel with each other, e.g., at the same time, using different wavelengths.

Out-of-band signaling coding includes subcarrier multiplexing (SCM) and dualwavelength coding. Packet header and payload in the SCM are transmitted in the same time slot, with header is placed at an electrical sub-carrier above the baseband frequencies occupied by payload. Packet header and payload are transmitted in the same time slot with separate wavelengths in the case of dual-wavelength coding.

\section{External Blocking}

In the packet switches, sometimes more than one packet try to go out through a similar 
output at the same time, thus creates blocking at the output interface. The solution is made as such that the only the permitted one is allowed to pass and the rest of the signals are buffered. Since this is a common problem for packet switches, three solutions are proposed: optical buffering, exploiting the wavelength domain, and deflection routing.

Optical Buffering - Optical Delay Lines (ODLs) or Fiber Delay Line (FDL) are the only technique to implement optical buffering. The ODL (or FDL) can make a packet wait for a specified amount of time. This delay time is related to the length of the delay line. However, delay lines are not commercially viable. The alternative is to convert the optical packet into electrical one, which is not viable since electronic memories can not match the speed of optical network. However, there are no suitable way to buffer the optical signals.

Exploiting Wavelength Domain - In the WDM, sometimes two packets are ready to be transmitted through the same output channel, creating blocking. One solution is that they are transmitted over two different wavelengths. This method requires that a single fiber combines number of wavelengths in it. The current coupling is about 200 wavelength/fiber.

Deflection Routing - This method is generally implemented for the switches that have little buffer space. Two conflicting output packets are routed as such that the correct one passes through the correct port. The other packet goes through available port and may result in longer delay or needed to be reordered at the destination. This process needs little or no buffering.

\section{Optical Packet Switching Architecture}

There are three optical packet switch architectures based on the switching fabric used were proposed in the literatures: space switch, broadcast-and-select, and wavelength routing.

\section{SPACE Switch}

The structure of the switch is $N \times N$ at both the input and output interface, as shown in the Fig.
1. Each of the fiber links has got $n$ wavelengths. Entire switch is slotted to facilitate the transmission of optical packet from the input to the output optical buffer. There are three parts in the switching fabric: optical packet encoder, space switch, and optical packet buffer.

The basic working principle of the switch is as follows: The optical packet encoder consists of optical demultiplexers and tunable wavelength converter (TWC). Demultiplexers split incoming optical signal to the $n$ different wavelengths. Each wavelength is fed to a TWC. TWC converts wavelength of the packet suitable for the destination output buffer.

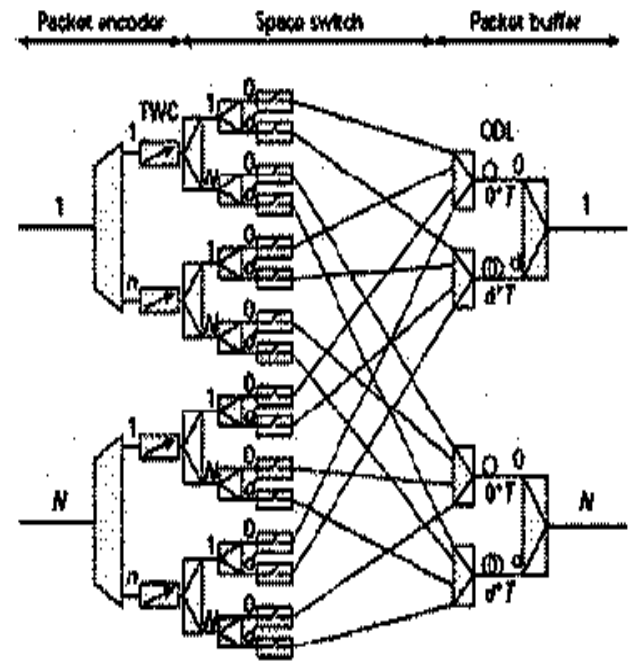

Fig. 1: Space Switch Architecture

Outputs of TWC are fed to splitters which distributes the same signal to each of the $\mathrm{N}$ different output fibers. Again these signals are separated into $d+1$ through another set of splitter. Output fibres from these splitters are connected to the ODLs, located at the destination output buffer. Then the packets are forwarded to appropriate optical gate, while rests are closed. The control unit possesses knowledge of entire switch. It may order a particular wave to be processed at a particular TWC and destine that to a particular FDL of the output buffer. 


\section{Broadcast-and-Select Switch}

All the packets from the input ports are combined on different wavelengths and broadcast to all the outputs. Wavelength selectors are used to select the output packet. Thus the switch itself leads to multicasting. There are two different architectures: KEOPS Switch with a Broadcast-and-Select, and Switch with Broadcast-and-Select and Recirculation Buffer.

KEOPS Switch with a Broadcast-and-Select - Originally developed as part of the European ACTS Keys to Optical Switching (KEOPS) project [5]. All input and output fibers carry single wavelength, as shown in Fig. 2. However, the wavelength of an output varies with packet. There are three blocks in the switch fabric: encoder, buffer, and selector.

Each of the encoder block consists of $N$ fixed wavelength converters (FWC) followed by a multiplexer. The buffer block consists of a splitter, $K$ ODL, and a space-switching stage implemented by means of splitters, optical gates, and combiners. Finally, the wavelength selector block consists of $\mathrm{N}$ wavelength channel selectors implemented by means of demultiplexers, optical gates, and multiplexers. These three blocks make up the broadcast-andselect switch fabric.

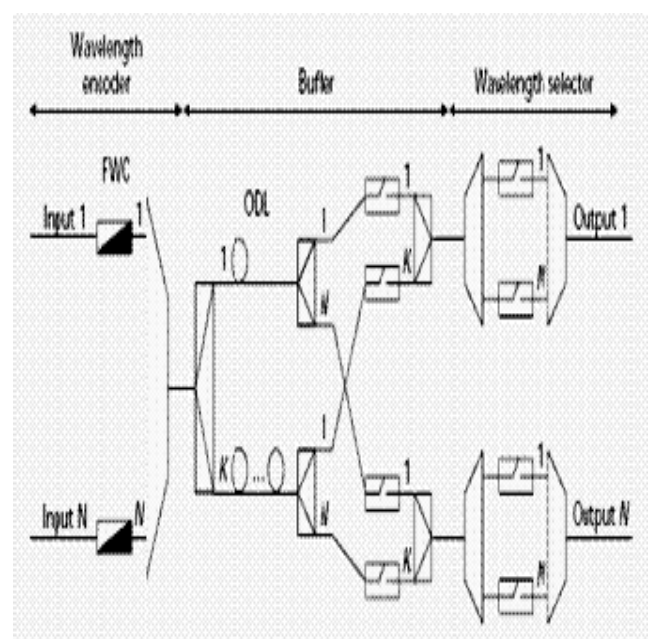

Fig.2: KEOPS Switch with a Broadcast-andSelect

The switch consists of header, payload and guard time. Header holds information about the packet and data is packet in the payload. The guard time is a special function that determines the setup time in the switch usually inserted either between the data and header or between two consecutive packets of the same wavelength. Also mixed rate coding is used where header is processed at a lower speed (in $\mathrm{Gb}$ scale) and the payload is processed at a higher speed (in Mb scale). A fixed time length in micro seconds is kept for the packets where the packet sizes are variable.

Thus the advantages are of two fold: the processing speed depends on the header rate not on the payload; and, buffering space does not depend on the payload rate. In the case of large scale deployment, the switch itself is considered a large one and the incoming signals are demultiplexed into a group of wavelengths which are in turn put to a similar number of group of wavelengths to be combined for transmission on a single output channel.

Switch with Broadcast-and-Select and Recirculation Buffer - This switch [6] was developed from a previous ATM switch, starlite. All input and output fibers carry single wavelength, as shown in Fig. 3. The output wavelength varies with packet. The switch is implemented with a coupler that couples incoming wavelengths through tunable wavelength converter (TWC) and distributes through tunable optical filters (TOF) and fixed optical filters (FOF). A control unit controls this slotted switch.

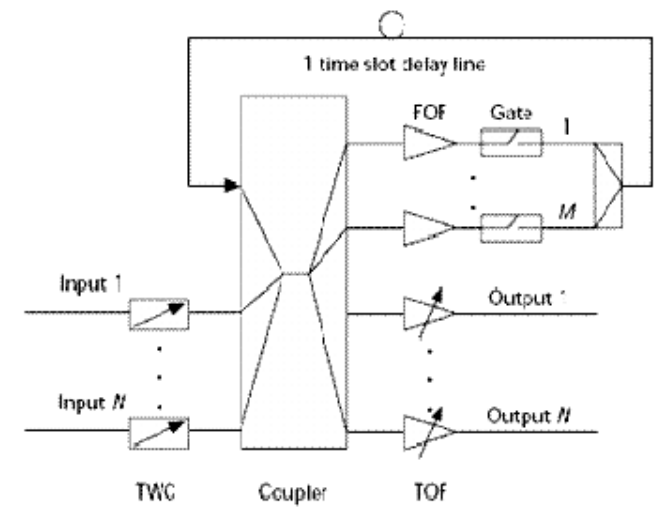

Fig. 3: A switch with broadcast-and-select fabric and recirculation buffer.

During a time slot, the control unit sends one incoming packet to the designated output along 
the single time slot delay. Those not going to proper destinations are recirculated through the ODL (FDL) and fed back the coupler in the following time slot.

7. Wavelength Routing Switch Architectures There are three different architectures for wavelength routing technique, which have two phases in their operations: ODL are used to solve contention; then packets are routed to output using wavelength routing. Those switching architectures are: input buffer, input buffer with a distribution network, and WASPNET.

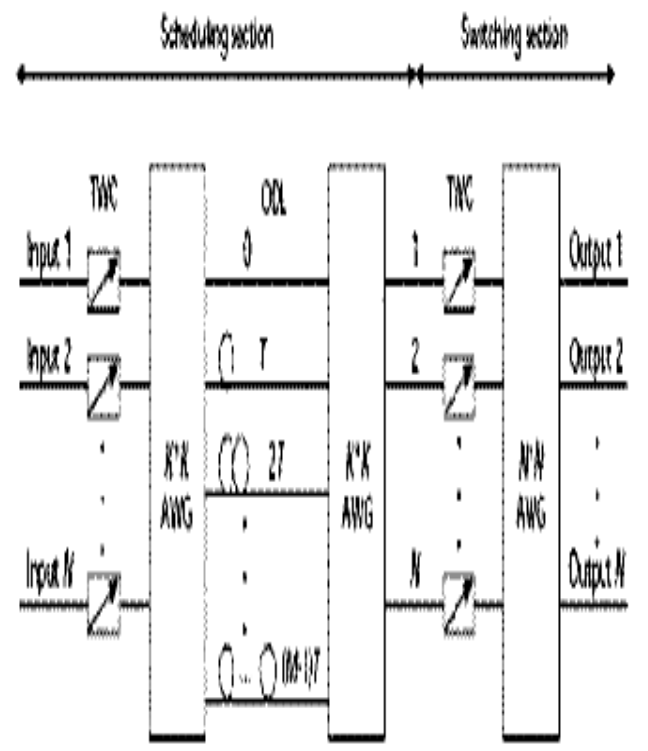

Fig. 4: An input-buffered switch.

\section{Input Buffer Switch}

There are switching and scheduling sections in this switch [7], as shown in fig 4. Incoming and outgoing ports carriy single wavelengths. Output wavelength varies with packets. Scheduling section is used to solve contentions though TWC at the inputs, then passes the packets through various arrayed waveguide gratings (AWG) and ODL in different time slots.

The delay rule for this switch: for a single time slot, outputs can not receive two similar packets; buffer output can not transmit two packets at the same time. The switching section is made up of AWG and TWC. Thus this section switches optical packets to their assigned outputs. However, one disadvantage of this switch is that it may suffer from headof-line blocking.

\section{Input-Buffered Switch with a Distribution Network}

This switch was developed as part of the KEOPS project [5], Fig 5. The output wavelength varies with packets. It also has contention resolution and switching parts. At the beginning demultiplexers pass packets to the designated ODL which is predetermined in the TWC upon the packets' entry and subsequent switching is implemented and then sent to the precise output.

Except for the distribution and input buffer at the input - which distributes the incoming packets to buffers - the rest of the switch is similar to the switches described earlier. The distribution part solves head-of-line blocking.

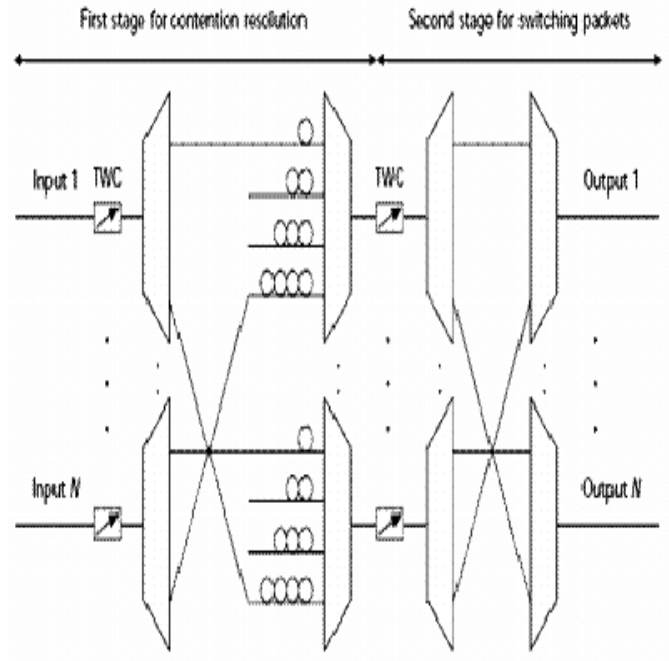

Fig. 5: An input-buffered switch with a distribution network.

\section{WASPNET Switch}

Wavelength Switch Optical Packet Network (WASPNET) is shown in Fig 6.

The switch [8] consists of TWC on both sides of the AWG. The first set of $N$ TWC for the incoming packets on the left of the AWG 
selects packets to be recirculated; the rest of the $N$ TWC converts packets to wavelengths for the output as required by the switch, where more packets remain than the incoming and outgoing packets. One advantage is that it can support priority for the optical packets through delay to allow any other high priority packet.

\section{Optical Burst Switching}

Optical Burst Switch (OBS) adapted International Telecommunication Union Telecommunication Standardization Sector (ITU-T) standard for burst switching in ATM networks, known as ATM block transfer (ABT). There are two versions of ABT: ABT with delayed transmission and ABT with immediate transmission. When a source wants to transmit a burst, it sends a packet to the ATM switches on the path of the connection to inform them that it wants to transmit a burst. If all the switches on the path can accommodate the burst, the request is accepted and the source is allowed to go ahead with its transmission.

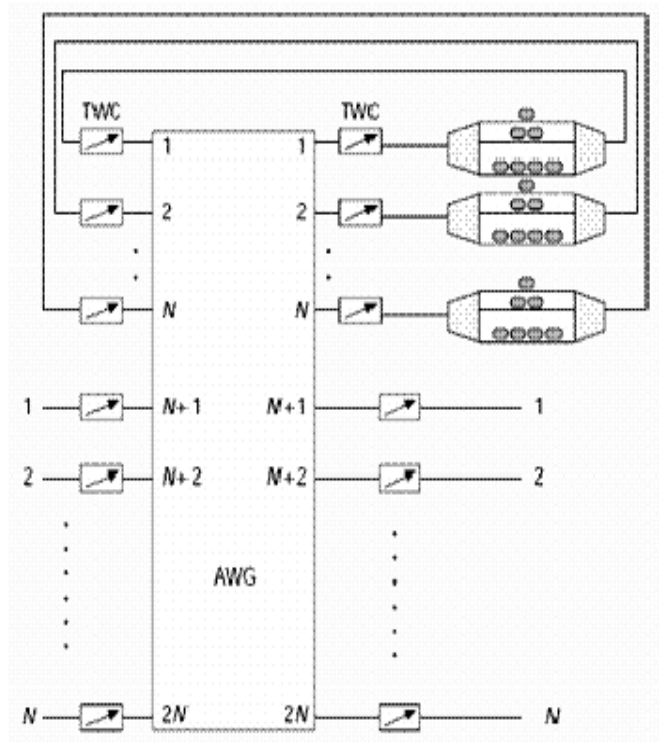

Fig. 6: The WASPNET switch.

Optical burst switches separate control and data payloads into two, allotting a channel for cooperatively small control signals over the intermediate nodes. These signals pass conventional $\mathrm{O} / \mathrm{E} / \mathrm{O}$ conversion, while the data payload is processed optically. The switch also uses offset time for the processing delay between the control and data signals. For a shorter offset time, the data burst is directly processed over the intermediate nodes and transmitted all optically without storing it in FDLs.

The fig 7, [9] shows the channels of the OBS. Only few control channels go through the $\mathrm{O} / \mathrm{E} / \mathrm{O}$ conversion. Thus FDLs are not deployed for data bursts in the switching fabric. Unless the switch operates in slotted manner, no synchronization is required. Moreover, FDLs and wavelength converters can help in burst loss [10]. Now the challenge is to operate the switch at a switching speed of the order of nanoseconds.

\section{Burst Assembly}

Burst assembly is the process of assembling incoming data from the higher layer into bursts at the ingress edge node of the OBS network. As packets arrive from the higher layer, they are stored in electronic buffers according to their destination and class. The switching unit forwards incoming packets to burst assembly units.

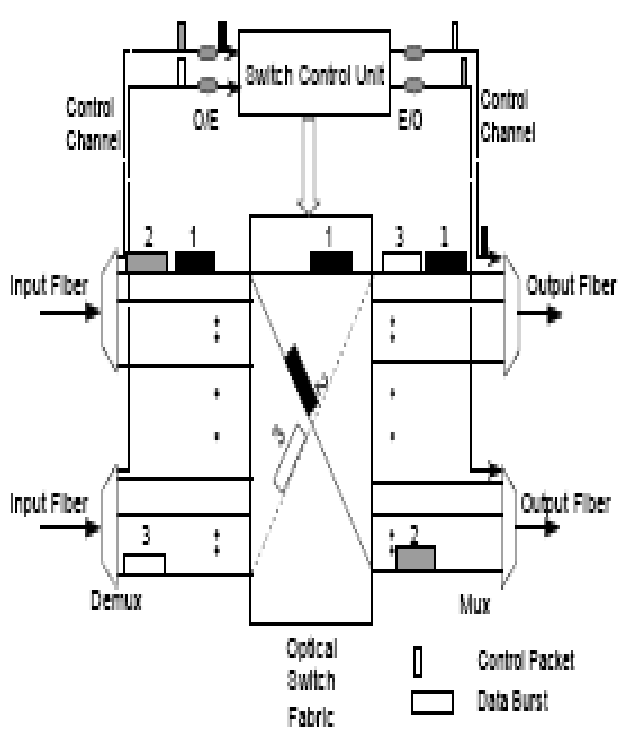

Fig. 7: Optical Burst Switching

The burst scheduler creates burst and their corresponding control packets, adjust offset 
time for each burst, schedule each burst on each output link and forward bursts and their control signals to the core network [11]. Recent studies show that different assembly schemes affect the assembled bursts traffic's characteristics [12, 13].

\section{Assembly Algorithms}

There are three classifications of assembly algorithms: timer-based, burt-slength-based, and mixed timer-burst-length. In the timerbased scheme, the timer starts at a new assembly cycle after a fixed time T. Packets arriving at the egress are combined into a burst. The time-out value is set carefully: if the value is too large, the packets will arrive at an intolerable time; if the value is too short, many small bursts will be generated and make overhead complex. However, the burst length is not guaranteed. The size of a burst is equal to the sum of the size of all the packets arriving in a fixed time period. Also the burst's interarrival time is constant.

For a burst-length-base scheme, a predetermined minimum burst length is considered. With new incoming bursts, the length of the already stored packets exceeds the minimum length. In this scheme, there is not prediction of assembly delay time. This has a Gaussian distribution characteristic. The variance of inter-arrival time of bursts that come from different edge nodes may become small with heavy traffic loads. This result in unwanted collisions of bursts and generates extra offset time losing synchronization.

Another way to achieve the both discussed in the above is through mixed-timer-burst-length scheme $[11,13]$. This is implemented with a specific burst length or a fixed timeout threshold.

Adaptive assembly algorithms - explore either the time threshold, burst length threshold or both adjusted dynamically. This algorithm has greater operational complexity though shows better performances with strongly correlated input packet traffic. Bursts generated in the adaptive assembly algorithm are buffered in the queue for an offset time while the control packet clears the path for the data signals in advance.

\section{Burst Reservation Protocol}

For the ATM networks, there are two burst level admission control mechanisms [14]: telland-go (TAG) and tell-and -wait (TAW). When tell-and-go (TAG) is used, the source transmits bursts without making any bandwidth reservation in advance. Sometimes bursts need to wait at the intermediate nodes until the forward path is cleared off. If such a path is not created, the signal is dropped off, or arrives at a later time than the anticipated time of arrival. This forms the basis for Tera-bit burst switching [15].

However, tell-and-wait (TAW), the source reserves a path up to the destination with a short request message prior to transmission. The intermediate nodes reserve a specific output link. This is informed to the source when the entire path is reserved. Only then the source starts sending the burst. If the path is not reserved, the source is notified and it dropped sending the burst.

If compared for propagation delay and burst length, tell-and-wait (TAW) outperforms telland-go (TAG). On the contrary, TAG is better when propagation delay is significant to the burst length.

A variant of tell-and-wait (TAW) is Just-intime (JIT) [16]. Just-in-Time (JIT) means that by the time a burst arrives an intermediate node, the switching fabric has already been configured, as shown in the fig 8 . In this scheme each burst transmission request is sent to a central scheduler. The scheduler then informs each requesting node the exact time to transmit the data burst.

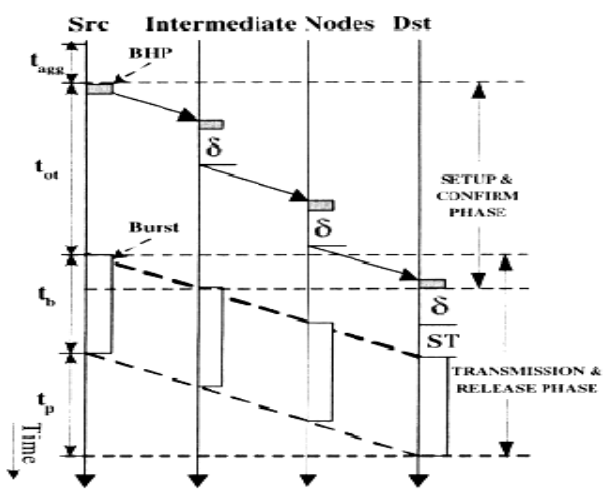

Fig.8. JIT protocol 
Another version of the JIT protocol is Just Enough Time (JET) [17, 18], based on hop-byhop reservation. This is one of the prevailing distributed protocols for OBS network. This does not require any kind of optical buffering or delay at each intermediate node. Each control packet carries the offset time information and makes a delayed reservation for the corresponding burst at the expected arrival time of the burst. The bandwidth is reserved for the first burst starting from the burst arrival time. At each intermediate node, the offset time is reduced to compensate for the actual control packet processing switch configuration time.

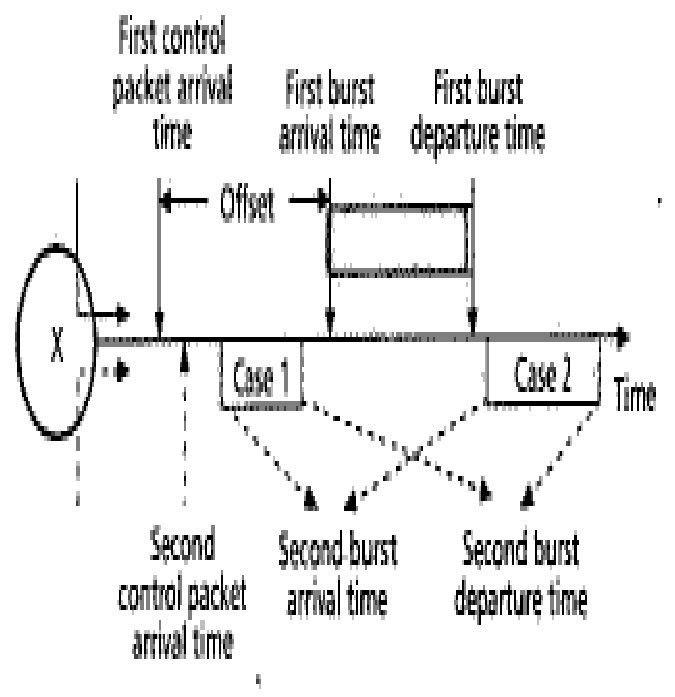

Fig. 9. JET protocol [18]

The effect of offset time varies with the deflection routing in the OBS network. If the burst takes a longer path, the minimal offset time for the primary path might not be enough. The burst length information is carried by the control packet. This enables to reserve a closeended reservation for the burst duration and an automatic release at the end. The intermediate nodes can make intelligent decisions on the possibility of such a reservation for a new burst and increase bandwidth utilizations.

\section{Burst Switching}

Unlike the electronic switches or routers, OBS employs no or limited buffer. The burst scheduling and connection are done in a different manner.

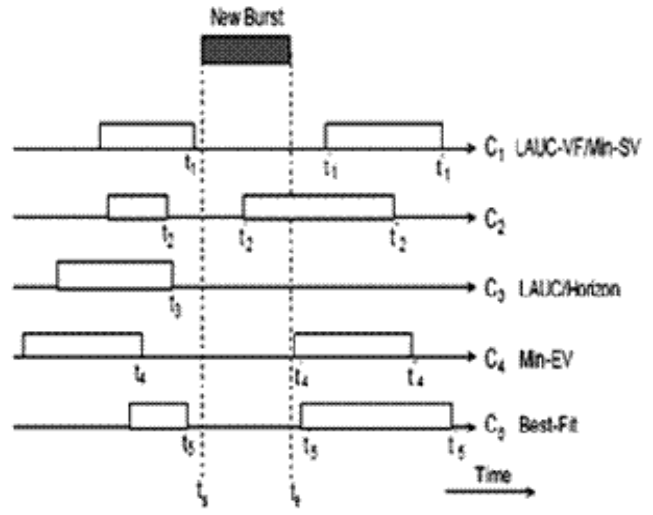

Fig. 10: A burst scheduling algorithm

\section{Scheduling Algorithms}

A simple burst scheduling algorithm is done, as in the fig 10. This is called Horizon or Latest Available Unscheduled Channel (LAUC) [19].

A single scheduling horizon is maintained for each wavelength. Only the channels with latest scheduling horizon is chosen, and scheduling horizons preceding the new burst are considered available. The horizon is then updated and makes reservation for the next burst. This algorithm eliminates minimum bandwidth gaps (or voids) due to the creations of new reservations. Such voids are generated when offset-time-based QoS or FDL sets are used. LUAC with void filling (LUAC-VF) is proposed [19]. This corresponds to the C1channel in the fig 10.

There are several classification of LUAC-VF: Min-SV (starting void), Min-EF (ending void), and best fit [20]. Min-SV is similar to LUACVF that employs techniques of computational geometry and is faster than LUAC-VF. MinEF minimizes newly created voids. The Best Fit minimizes the total length of starting and ending voids, generated after the reservation.

\section{Contention Resolution}

In a bufferless OBS network, contention among bursts can be resolved in three ways: deflection, dropping, and preemption.

Contention usually occurs when bursts try to occupy the same wavelength at the same time. Deflection sends a burst to another output 
channel when it tries to make a contention. This is achieved through wavelength, space or/and time domain.

A contending burst is sent to another wavelength through wavelength conversion in the wavelength domain. A contending burst can be sent to a different port and then follow a different rout through the space domain [18]. Employing FDL (or ODL), a contending burst can be delayed using time domain [20]. However, in the case when a contending burst can not be deflected in any of the mentioned means, then the burst is dropped. An incoming burst can preempt an existing burst based on priority or traffic profile. Another approach, called burst segmentation [21, 22] breaks incoming bursts or existing bursts into multiple segments which may be deflected, dropped or preempted.

Sometimes contention schemes can be applied simultaneously. During contention, a burst is not simply forwarded to an alternate route, rather pass the burst back to the node where contention occurred, and then transmit is again through the correct path. This makes the network work like an ODL (or FDL). This is done in expense of time delays.

If wavelength conversion is not applied, burst loss performance statistics may be calculated to evaluate bursts whose loss rates are minimal. These special bursts are given higher priority every time they enter those nodes [23].

\section{An Optical INTERNET}

Most of the literatures were dedicated to the QoS provisioning in the Internet. However, they could not come out of the classical concepts of electrical packet switching and buffers. Though the FDL was mentioned in many literatures, however, FDLs are not viable for providing with delays for the optical data. Three different approaches were proposed for service differentiation.

Firstly, offset-time-based differentiation is easy to implement. Sufficiently large offset time is allotted to high priority bursts. This also introduces additional delay at the edge. The performance of the differentiation depends on the burst length and interarrival time distributions [18]. Active dropping can avoid above mentioned shortcomings.

Secondly, selected dropping of bursts is initiated according to loss rate measurement or traffic profile to guarantee the high-priority class to make a successful reservation. Both of these two schemes make differentiation at the burst level. Third one, which works at the packet level, can be achieved with burst segmentation. This mixes up low-priority and high-priority packets in special manner: putting low-priority bursts at the head and tail and high-priority bursts in the middle. If packets at the head or tail are dropped while in contention, differentiation on packet loss can be achieved [22].

\section{IP / WDM Multicast}

Current and emerging Internet applications should be efficiently supported through multicast. A straightforward way to do multicasting in an OBS network is Separate Multicast in which multicast traffic and unicast traffic are assembled separately into different bursts. The Tree-Shared Multicasting scheme was proposed [24], that reduces the overhead due to guard bands and the control packet associated with each burst. This was also researched whether two multicast sessions could share a tree. If done in this manner, one of the benefits will be that a burst would be delivered to unintended destinations via a shared multicast tree. The other benefit would be the degree of overlapping among the multicast sessions through sharing strategy of the multicast tree.

\section{TCP over OBS}

Since TCP is the mostly used data transmission protocol, it is envisaged that this will become the backbone for the future Internet backbone. Therefore TCP over OBS is of much interest in the research arena. One study [28] found that TCP throughput decreases with burst assembly process that in fact increases the round trip time. One of the consequences is that a TCP 
source with relatively low access bandwidth in the local IP access network and small burst assembly time at the edge can have only one TCP segment in one burst. The second consequence is that there is no correlation gain. However, the one benefit is related to insignificant delay penalty, which provides with similar throughput without burst assembly. Still there is a shortfall when all high access bandwidths with long bursts from a TCP source are assembled into one burst, it makes correlation gain maximum and large delay penalty.

In their recent studies, Yang Chen et al., found that for a TCP source that has a medium access bandwidth (between low and high relative to the burst assembly time), using an adaptive assembly algorithm yields the best throughput because it can adjust the assembly time to match the TCP congestion control mechanisms.

\section{LOBS}

A natural extension of the generalized multi protocol label switching (G-MPLS) in OBS networks, labeled OBS (LOBS), was proposed in [25]. LOBS let OBS to control packet carrier to carry additional label information. Label and wavelength are interconnected on time scale, but not on burst. This makes subwavelength and statistical multiplexing possible. A major benefit is that LOBS is based on TCP protocol, switches all data alloptically, and facilitates seamless integration of IP and WDM. However, bursts belonging to the same LOBS path can be sent on different output wavelengths, or can interleave on the same wavelength meaning that different bursts may be merged into one LOBS path.

\section{Qualitative Comparison of OPS and OBS} Basic switching node architectures were discussed in the preceding texts. It is clear that all-optical switching will not be economically viable in the near future due to technical constraints, the optical packet switching (OPS) approach will likely to remain the only choice of faster transmission backbones. It is observed that for a 1 millisecond delay, 200 kilometers of fiber is required.

Though recent advancements in semiconductor optical amplifiers (SOA) are observed [22], still it will take some more time before OBS could be used in the transmission backbones.

A comparison between optical packet switching (OPS) and the optical burst switching (OBS) is illustrated in the Table 1.

According to the comparison shown in Table 1 , it is observed that the procedures for processing and synchronization of the overhead for OBS are better than the OPS. The main advantage, as we also discussed in the text, was that the OBS do not require any optical buffer for storing data in the switching node.

\section{Concluding Remarks}

The difference between optical packet switching (OPS) and optical burst witching (OBS) was discussed in the article. The concepts were discussed comparing with existing optical switches. However, the article did not show the economical aspects for the OBS. The paper tried to comprehend various researches those were done in the past.

Both the OPS and OBS are in the experimental stage. One of the draw back in developing commercially viable optical packet switches is that there are no effecting optical buffers. Also IP packet transmission is required to be solved, regarding the size, memory capacity, nature of the opto-electric interfaces. Though optical burst switches seem more appropriate for commercial use, no optical buffering should be included. This type of switches may be initially deployed at the metropolitan rings to connect IP routers.

Various models and algorithms were discussed. The article showed that there are many works left for the researchers to develop OBS. 
Table 1: [4]

\begin{tabular}{|c|c|c|c|c|}
\hline $\begin{array}{c}\text { Bandwidth } \\
\text { utilization }\end{array}$ & $\begin{array}{l}\text { Latency } \\
\text { (set-up) }\end{array}$ & Optical buffer & $\begin{array}{c}\text { Proc./Sync. Overhead (per } \\
\text { unit data) }\end{array}$ & $\begin{array}{c}\text { Adaptively } \\
\text { (traffic and fault) }\end{array}$ \\
\hline \multicolumn{5}{|c|}{ Optical Packet Switching (OPS) } \\
\hline high & low & required & high & high \\
\hline \multicolumn{5}{|c|}{ Optical Burst Switching (OBS) } \\
\hline high & low & Not required & low & high \\
\hline
\end{tabular}

\section{References}

[1] C. A. Brackett, "Dense wavelength division multiplexing networks: Principles and applications," IEEE J. Select. Areas Commun., vol. 8, pp. 948-964, Aug. 1990.

[2] B. Mukherjee, Optical Communication Networks. New York: McGraw-Hill, 1997.

[3] Lisong Xu, Harry G Perros, George Rouskas, "Techniques for Optical Packet Switching and

Optical Burst Switching,” IEEE Communications Magazine, January 2001, pp 136-142.

[4] Yang Chen, Chunming Qiao and Xiang Yu, "Optical Burst Switching: A New Area in Optical Networking Research,” IEEE Network, May/June, 2005, pp 16-23

[5] C. Guillemot et al., "Transparent Optical Packet Switching: The European ACTS KEOPS Project Approach,” J. Lightwave Tech., vol. 16, no. 12, Dec. 1998, pp. 2117-33.

[6] G. Bendeli et al., "Performance Assessment of a Photonic ATM Switch Based on a Wavelength Controlled Fiber Loop Buffer,” OFC'96 Technical Digest, OFC, 1996, pp 106-7.

[7] W. D. Zhong and R. S. Tucker, "Wavelength Routing-Based Photonic Packet Buffers and Their Applications in Photonic Packet Switching Systems,” J. Lightwave Tech., vol. 16, no. 10, Oct. 1998, pp. 1737-45.

[8] D. K. Hunter et al., "WASPNET: A Wavelength Switched Packet Network," IEEE Commun. Mag., Mar. 1999, pp. 120-29.

[9] Byrav Ramamurthy, "Wavelength Conversion in WDM Netwroking," IEEE Journal on Selected Areas in Comm., vol. 16, no. 7, Sep 1998,pp 1061-1073

[10] C. Gouger, "Contention Resolution in Optical Burst Switching Networks," Advanced Infrastructures for Photonic Networks, WG 2 Intermediate Report, 2002. pp. 62-82.

[11] Y. Xiong, M. Vandenhoute, and H. Cankaya, "Control Architecture in Optical Burst switched WDM Neworks," IEEE JSAC, vol 8, 18 Oct 2000, pp. 1838-51

[12] K. Laevans, "Traffic Characteristics Inside Optical Burst Switched Network," Proc. Opticomm, 2002, pp. 137-48.
[13] X. Yu, Y. Chen, and C. Qiao, "Study of Traffic Statistics of Assembled Bunt trafic in Optical Burst Switched Network,” Proc. Opticomm, 2002. pp. 149-59.

[14] I. Widjaja, "Performance Analysis of Burst Admission-control Protocols," IEE Proc. Commun., vol. 142, Feb 1995, pp 7-14

[15] J. Turner, “Terabit Burst Switching,” JHS, vol. 8, no. 1, 1999, pp. 3-16.

[16] D. L. Mills, et al., "Highball: A High Speed, Reserved-Access, Wide-Area Network," Tech. rep. 90-9-3. Elm. Eng. Dept., Univ. of Delaware, 1990

[17] M. Yoo and C. Qiao, "Just-Enough-Time (JET): A High Speed Protocol for Bursty Traffic in Optical Networks,” Prc. IEEE/LEOS Conf. Tech. Global Info. Infrastructure, Aug. 1997, pp. 26-27.

[18] C. Qiao and M. Yoo, "Optical Burst Switching (OBS) A New Paradigm for an Optical Internet," JHS, vol. 8, no. 1, 1999, pp. 69-84.

[19] Y. Xiong, M. Vandenhoute, and H. Cankaya, "Control Architecture in Optical Burst switched WDM Neworks," IEEE JSAC, vol 8, 18 Oct 2000, pp. 1838-51

[20]J. Y. Wei and R. J. McFarland, "Just-In-Time Signaling for WDM Optical Burst Switching Network,” JLT, vol. 18, no. 12, Dec. 2000, pp 2019-37

[21] V. M. Vokkarane and J. P. Jue, "Segmentationbased Non-Preemptive Scheduling Algorithms for Optical Burst-Switched Networks,” Proc. 1st Int'l. Wksp, OBS, Oct 2003

[22] V. M. Vokkarane and J. P. Jue, "Prioritized Burst Segmentation and Composite BurstAssembly Techniques for QoS Support in Optical Burst-Switched Networks," IEEE JSAC, vol. 21, no. 7, 2003, pp 1198-1209

[23] X. Wang, H. Morikawa and T. Aoyama, "Priority-based Wavelength Assignment Algorithm for Burst Switching Photonic Network,” Proc. OFC, 2002, pp 765-67

[24] M. Jeong, H. Cankaya and C. Qiao, "On a New Multicasting Approach in Optical Burst Switched Networks,” IEEE Commun. Mag., Nov. 2002, pp 96-103

[25] C. Qiao, "Labeled Optical Burst Switching for Ip-Over-WDM Integration,” IEEE Commun. Mag., Sept. 2000, vol. 38, no. 9, pp 104-14 